\title{
El video tutorial como recurso para su aplicación en la metodología docente "flipped classroom" dirigida a alumnos de postgrado en Bellas Artes
}

\section{Gloria Lapeña Gallego ${ }^{a}$ y José Mayor Iborra ${ }^{b}$}

aDepartamento de Dibujo. Facultad de Bellas Artes. Universidad de Granada. lapena@ugr.es bDepartamento de Bellas Artes. Facultad de Bellas Artes. Universidad de Murcia. jmayor@um.es

\begin{abstract}
The master's degree in Fine Arts is a preliminary step for those students who intend to complete a PhD. For this reason, the programme includes a compulsory subject whose objective is the acquisition of the competences related to research. One of the difficulties that the students usually find in this subject concerns the location of quality bibliographic sources on which to base their research. In the present work we set out our teaching innovation project, which consists of the elaboration and publication of four video tutorials about the bibliographic data sources. The access to these videos by the students of the subject of the master's degree "Methodology and research in Fine Arts" allows us the application of the teaching methodology known as "flipped classroom" and encourages self-directed learning. In this way, class hours can be used for personalized guidance by the teacher.
\end{abstract}

Keywords: flipped classroom, teaching methodology, Fine Arts, postgraduate studies.

\begin{abstract}
Resumen
El máster universitario en Bellas Artes es un paso previo para el estudiante que tiene previsto realizar una Tesis Doctoral. Por este motivo, sus programas incorporan una asignatura obligatoria cuyo objetivo es la adquisición de competencias relacionadas con la investigación. Una de las dificultades que encuentran los estudiantes de esta materia es localizar y seleccionar fuentes bibliográficas de calidad en las que basar su investigación teórica. En el presente trabajo resumimos nuestra propuesta de innovación docente, consistente en la elaboración y publicación en acceso abierto de cuatro video tutoriales sobre sendos catálogos de fuentes de datos bibliográficos. El acceso a estos videos por parte del estudiante de la asignatura de máster "Metodología e investigación en Bellas Artes" permite la aplicación de la metodología docente "flipped classroom" y favorece el aprendizaje autónomo. De este modo, las horas presenciales son aprovechadas para la orientación personalizada por parte del profesor.
\end{abstract}

Palabras clave: flipped classroom, metodología docente, Bellas Artes, estudios de postgrado. 


\section{Introducción}

El Máster Universitario en Producción y Gestión Artística, el único impartido en la Facultad de Bellas Artes de la Universidad de Murcia (UM), desarrolla dos líneas de intensificación: la de la producción y la de la gestión artística. Salvo dos asignaturas obligatorias, "Metodología e Investigación en Bellas Artes” y “Trabajo Fin de Máster”, el estudiante diseña su itinerario mediante la elección de ocho asignaturas entre las dieciséis ofertadas. Estos estudios, además de completar la formación de los cuatro años del Grado en Bellas Artes, permiten el acceso al doctorado, por lo que contribuyen a una actividad de iniciación a la investigación.

Tal y como consta en la Guía Docente (https://www.um.es/web/bellasartes/), en la asignatura "Metodología e Investigación en Bellas Artes" se fundamentan los territorios acerca del qué se debe investigar en las Bellas Artes desde la Universidad. Su carácter de obligatoriedad se debe a la presentación al estudiante de los instrumentos necesarios para abordar, construir, enfocar y sistematizar metodológicamente un trabajo de investigación en el resto de las asignaturas del Máster (incluido su TFM) y en su futura Tesis Doctoral.

La asignatura se estructura en dos partes diferenciadas. Una de ellas se centra en el análisis de textos críticos, y la otra, de la cual nos ocupamos en este estudio, aborda las condiciones de la investigación científica y los métodos y estrategias para establecer los fundamentos teóricos de la creación artística. Cualquier estudiante matriculado en la UM tiene acceso a la Biblioteca digital de esta Universidad, donde puede consultar en catálogos, en libros electrónicos, en revistas y en bases de datos. Además, si los estudios en los que se encuentra matriculado son de máster o de doctorado, puede acceder a información a través de la plataforma dinámica Web of Science (WOS). Esta opción le permite consultar todas las bases de datos al mismo tiempo y obtener textos completos de artículos que puedan interesarle para elaborar su trabajo de investigación.

El acceso a estos recursos, su análisis, tratamiento y organización se encuentran documentados de manera general en video tutoriales de la Biblioteca. Sin embargo, la formación específica para los alumnos de este Máster a través del Aula Virtual requiere una atención especial dadas las connotaciones de la investigación en las Humanidades, y más concretamente en las Artes. Tras la aprobación del Plan de Bolonia, las enseñanzas universitarias en Bellas Artes no se orientan exclusivamente a la producción artística como si se tratase de una diplomatura superior, sino que la formación implica además la investigación académica (García Fortes, 2007). El futuro artista es también un intelectual transmisor de conocimiento, y para ello se nutre de fuentes bibliográficas de calidad que abarcan diferentes disciplinas.

En el presente texto resumimos el proyecto de innovación docente realizado para la asignatura "Metodología e Investigación en Bellas Artes" del Máster en Producción y Gestión Artística. La acción se concreta en la publicación en acceso abierto de cuatro video tutoriales que facilitan el planteamiento de proyectos artísticos fundamentados en el conocimiento y en la capacidad crítica de los estudiantes de postgrado en Bellas Artes. La publicación de estos video tutoriales previa a la impartición de la asignatura permite el 
aprendizaje autónomo y el aprovechamiento de las horas presenciales para la orientación personalizada por parte del profesor. Esta dinámica de aprendizaje se encuadra dentro de la metodología docente conocida como "clase invertida" o "flipped classroom" (Bergman y Sams, 2014). El proyecto de innovación estuvo soportado por la Unidad de Innovación de la Universidad de Murcia (curso 2015/2016) y asesorado por la responsable de la Hemeroteca Científica de esta misma Universidad.

\section{Objetivos}

Distinguimos dos tipos de objetivos: los objetivos generales, relacionados con la naturaleza del material a producir, y los objetivos específicos, vinculados a los contenidos del material en base a los recursos ya existentes en la UM.

\subsection{Objetivos generales}

- Implementar, en el Aula Virtual de la UM, la asignatura "Metodología e Investigación en Bellas Artes" dentro del Máster Universitario en Producción y Gestión Artística mediante video tutoriales adaptados a las líneas de investigación propias del Área.

- Facilitar a los estudiantes de este Máster la utilización de recursos digitales para la búsqueda de información en la que basar sus procesos creativos con anterioridad a la clase magistral presencial.

\subsection{Objetivos específicos}

- Conocer los recursos electrónicos de investigación de la Universidad de Murcia y manejar los recursos del Catálogo Alba de la BUMU.

- Saber acceder a los recursos restringidos a investigadores, a través del escritorio virtual EVA o por configuración del Proxy, e identificar los que pertenecen a las áreas de las Bellas Artes.

- Realizar de manera autónoma diferentes búsquedas bibliográficas relacionadas con la línea de investigación personal de cada estudiante.

\section{Desarrollo de la innovación}

El trabajo de innovación requiere de una planificación antes de proceder a la elaboración del material para alojar en https://tv.um.es/ con el fin de no duplicar los tutoriales ya existentes en la UM. En primer lugar analizamos las necesidades en cuanto a material bibliográfico de los ocho alumnos matriculados en el curso en el que desarrollamos el proyecto (2015/2016) en el Máster de Producción y Gestión Artística. Para ello elaboramos una encuesta de catorce preguntas con cuatro opciones que, gradualmente, van desde "totalmente de acuerdo" hasta "en desacuerdo". En un primer grupo de siete preguntas, valoramos el grado de utilización por parte de cada estudiante de recursos de búsqueda como Google, la hemeroteca de la Universidad de Murcia, el idioma de preferencia y la bibliografía recomendada en las guías docentes. El otro grupo de preguntas se centra en la temática en la que van a basar la investigación de su Trabajo Fin de Máster (TFM) y su 
intención de continuar sus estudios de doctorado una vez superado el Máster. A continuación, seleccionamos las revistas digitales accesibles relacionadas con las tres áreas de las Bellas Artes (Dibujo, Pintura y Escultura) y las vías de acceso a las mismas. Por último, grabamos y editamos los video tutoriales y los alojamos en el canal abierto de la UM (https://tv.um.es/). Realizamos una evaluación continua del proyecto por observación directa dado el número reducido de estudiantes y la completamos con encuestas en sucesivos cursos hasta la actualidad (finalizado el curso 2018/2019).

\section{Resultados}

Los resultados de la encuesta inicial realizada a los ocho alumnos matriculados en el curso 2015/2016 muestran que la mayoría realiza las búsquedas mediante la introducción de palabras clave en español en Google y acude a la biblioteca a consultar los libros recomendados en la guía docente. En relación a la búsqueda para la elaboración del TFM y su posible continuidad en los estudios de postgrado (segundo grupo de cuestiones), prácticamente todos los alumnos habían acotado su línea de investigación y manifestaron su decisión de iniciar el doctorado, si bien solamente uno de ellos tenía avanzada su investigación teórica.

El grado de satisfacción del proyecto desarrollado, dado el bajo número de estudiantes en este Máster, la realizamos mediante observación directa durante las clases semanales. Hemos encontrado una aceptación de los recursos creados, quedando constancia en el elevado número de reproducciones de los videos a pesar del bajo número de matrículas en postgrado de Bellas Artes de la Universidad de Murcia.

En cuanto a la elaboración del material digital para su utilización en la "clase invertida" del curso 2015/2016 y sucesivos, describimos los contenidos de cada base de datos de recursos digitales, referimos el enlace del video tutorial elaborado y alojado en el canal tv.um.es dentro de la Serie: "Metodología e Investigación en Bellas Artes" e indicamos el número de reproducciones desde la fecha de su alojamiento (octubre de 2016) hasta la actualidad (marzo de 2019).

\subsection{Catálogo Alba}

Catálogo compuesto por registros bibliográficos físicos que se encuentran en cualquiera de las Bibliotecas de la Universidad de Murcia. Se puede acceder a él desde cualquier ordenador conectado a Internet. Una vez localizado el recurso, se puede tomar prestado para su consulta con el carnet de estudiante de la Universidad de Murcia.

Video tutorial (671 reproducciones) disponible en http://tv.um.es/video?id=83251\&cod=a1

\subsection{Plataforma LEO}

Plataforma de préstamo de libros electrónicos y audiolibros para usuarios de la Universidad de Murcia. A diferencia de los préstamos físicos de cualquier biblioteca de la Universidad, 
no hay penalización por retraso en la devolución, pues cumplido el plazo de préstamo se devuelve automáticamente.

Video tutorial (482 reproducciones) disponible en: $\underline{\text { http://tv.um.es/video?id=83261\&cod=a1 }}$

\subsection{Catálogo Xabio}

Catálogo compuesto por registros bibliográficos físicos y electrónicos. Algunos de ellos son de pago, por lo que su acceso está restringido a profesores pertenecientes a grupos de investigación y alumnos matriculados en algún programa de máster o doctorado. El sistema de filtros por áreas, idioma, fecha de publicación, etc. permite afinar la búsqueda.

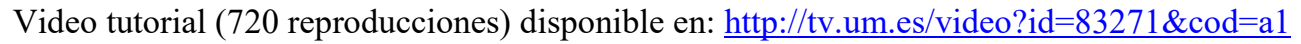

\subsection{Biblioteca del CENDEAC}

Centro de Documentación y Estudios Avanzados de Arte Contemporáneo perteneciente a la red de bibliotecas públicas de la Región de Murcia. Por medio de una tarjeta de identificación facilitada por la Biblioteca Regional se pueden tomar prestados documentos disponibles en cualquiera de estas bibliotecas.

Video tutorial (229 reproducciones) disponible en: $\mathrm{http}: / / \mathrm{tv}$. um.es/video?id=83281\&cod=a1

\section{Conclusiones}

Los recursos bibliográficos, tanto físicos en bibliotecas y hemerotecas de las universidades como digitales en red, son herramientas fundamentales en la investigación dentro de cualquier área de conocimiento. La Universidad de Murcia pone a disposición de los usuarios información acerca de cómo acceder y seleccionar dichos recursos. En una primera prospección observamos que los estudiantes del área de las Bellas Artes que inician su carrera investigadora de postgrado restringen sus búsquedas por introducción de palabras clave en Google y/o en los libros recomendados en las guías docentes. Ello se debe fundamentalmente a que los intereses del artista-investigador de postgrado pueden encuadrarse en áreas muy dispares que van desde las tecnológicas (Net.Art, arte sonoro) hasta las ciencias sociales (arte colaborativo, arte público) pasando por las ciencias experimentales (Ecoarte). La elaboración y publicación de material docente adaptado a los intereses específicos de estos estudiantes, nos ha permitido incentivar el trabajo autónomo previo a la clase presencial, pudiendo disponer de esta última para la tutoría personalizada.

\section{Referencias}

BERGMANN, J. y SAMS, A. (2014). Dale la vuelta a tu clase: lleva tu clase a cada estudiante, en cualquier momento y cualquier lugar. Madrid: S.M. 
El video tutorial como recurso para su aplicación en la metodología docente "flipped classroom" dirigida a alumnos de Postgrado en Bellas Artes

GARCÍA FORTES, S. (2007). "Los estudios de Bellas Artes en la Universidad de Barcelona ante el reto del Espacio Europeo Superior: de las especialidades a los títulos de Grado" en Observar, vol. 1, pp. 46-55.

LAPEÑA GALLEGO, G. "El Catálogo Alba". tv.um.es $<$ http://tv.um.es/video?id=83251\&cod=al $>$ [Consulta: 14 de marzo de 2019]

LAPEÑA GALLEGO, G. "La plataforma LEO". $t$ v.um.es $<$ http://tv.um.es/video?id=83261\&cod=a1 $>$ [Consulta: 14 de marzo de 2019]

LAPEÑA GALLEGO, G. "El Catálogo Xabio". tv.um.es $<$ http://tv.um.es/video?id=83271\&cod=al $>$ [Consulta: 14 de marzo de 2019]

LAPEÑA GALLEGO, G. "La biblioteca del CENDEAC". tv.um.es

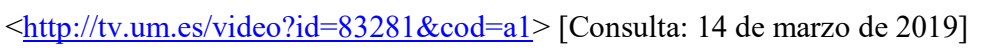

UNIVERSIDAD DE MURCIA. Guía Docente de la asignatura de Máster "Metodología e Investigación en Bellas Artes" 2018/2019 <https://aulavirtual.um.es/umugdocentetool/htmlprint/guia/RHVnsb1kdMYKPHLucenA71QCUjuRrRlGeHsxYE5bH321R13fNsy>

[Consulta: 14 de marzo de 2019] 